\section{Macrophytes Du Lac Iro Au Tchad : Diversité, Typologie Phytosociologique Et Pressions}

\section{Résumé}

\section{Goy Saradoum,}

Enseignant-chercheur, Maître-Assistant, Département de Biologie et Géologie, Faculté des Sciences et Techniques, Université de Sarh, Tchad

\section{Guiguindibaye Madjimbe,}

Enseignant-chercheur, Maître-Assistant, Département des Sciences Fondamentales, Faculté des Sciences Agronomiques et de l'Environnement, Université de Sarh, Tchad

\section{Kemkong Baouyé,}

Enseignant-chercheur, Assistant, Département de Biologie et Géologie, Faculté des Sciences et Techniques, Université de Sarh, Tchad

\section{Doloum Gomoung,}

Enseignant-chercheur, Maître-Assistant, Département de Biologie et Géologie, Faculté des Sciences et Techniques, Université de Sarh, Tchad

\section{Brahim Amba,}

Enseignant-chercheur, Assistant, Département de Développement des Ressources Halieutiques, Faculté des Sciences Agronomiques et de l'Environnement, Université de Sarh, Tchad

Submitted: 24 September 2020

Accepted: 24 November 2020

Published: 31 December 2020

Corresponding author:

Goy Saradoum

\section{DOI: 10.19044/esj.2020.v16n36p105}

(c)

Copyright 2020 Saradoum G,

Distributed under Creative Commons BY-NC-ND 4.0 OPEN ACCES

Cite as:

Saradoum G, Madjimbe G, Baouyé K, Gomoung D, Amba B.(2020). Macrophytes du lac Iro au Tchad : diversité, typologie phytosociologique et pressions. European Scientific Journal, ESJ, 16 (36), 1.

https://doi.org/10.19044/esj.2020.v16n36p105
Cette étude entreprise dans le lac Iro a portée sur l'estimation de la diversité macrophytique, l'identification des groupements végétaux, l'évaluation des potentielles menaces qui pèsent sur cette végétation et la détermination de la composition physico-chimique de l'eau. L'analyse des végétations a été faite par la technique des transects et la typologie des groupements végétaux par la méthode phytosociologique Braun-blanquetiste. L'estimation des indices de diversité et la discrimination des différents groupements végétaux ont été faites à l'aide du logiciel PAST. Plusieurs matériels ont été utilisés pour déterminer la qualité de l'eau. L'étude a permis de recenser 30 espèces reparties en 21 genres et 12 familles dont 96,42\% d'Angiospermes et de 3,58\% de Ptéridophytes. Les familles les mieux représentées sont les Poaceae, les Fabaceae et les Cyperaceae. Les espèces les plus fréquentes et présentes sur presque tous les transects sont : Echinochloa colona, Echinochloa stagnina, Neptunia oleracea, Ceratophyllum demersum et Pistia stratioites. Il a été distingué 6 groupements dont certains peuvent être intégrés dans des syntaxons existants dans d'autres écosystèmes lacustres. La qualité de l'eau est globalement favorable au développement des espèces aquatiques mais souvent polluée dans les parties très fréquentées. Les pressions qui s'exercent sur les phytocénoses du lac Iro témoignent de la vulnérabilité de cet écosystème.

Subject: Plant Ecology

Mots-clés: Macrophytes, Diversité Floristique, Groupements Aquatiques, Lac Iro. 


\section{Macrophytes from Lake Iro In Chad: Diversity, Phytosociological Typology And Pressures}

\section{Goy Saradoum,}

Enseignant-chercheur, Maître-Assistant, Département de Biologie et Géologie, Faculté des Sciences et Techniques, Université de Sarh, Tchad

\section{Guiguindibaye Madjimbe,}

Enseignant-chercheur, Maître-Assistant, Département des Sciences Fondamentales, Faculté des Sciences Agronomiques et de l'Environnement, Université de Sarh, Tchad

Kemkong Baouyé,

Enseignant-chercheur, Assistant, Département de Biologie et Géologie, Faculté des Sciences et Techniques, Université de Sarh, Tchad

\section{Doloum Gomoung,}

Enseignant-chercheur, Maître-Assistant, Département de Biologie et Géologie, Faculté des Sciences et Techniques, Université de Sarh, Tchad

\section{Brahim Amba,}

Enseignant-chercheur, Assistant, Département de Développement des

Ressources Halieutiques, Faculté des Sciences Agronomiques et de

l’Environnement, Université de Sarh, Tchad

\section{Abstract}

This study in Lake Iro was focused on estimating macrophytic diversity, identifying plant groups, assessing potential threats to this vegetation, and determining the physico-chemical composition of water. Vegetation analysis was done by transect technology and plant group typology by the Braun-blanquetiste phytosociological method. The estimation of various diversity indices and the discrimination of different plant groups were made using the PAST software. Several materials were used to determine water quality. There were 30 species distributed among 21 genera and 12 families, of which 96,42\% were Angiosperms and 3,58\% were Pteridophytes. The best represented families were Poaceae, Fabaceae and Cyperaceae. The most frequent and present species on almost all transects were : Echinochloa colona, Echinochloa stagnina, Neptunia oleracea, Ceratophyllum demersum and Pistia stratioites. There were 6 groupings, some of which can be integrated into existing syntaxons in other lake ecosystems. Water quality was generally favorable to the development of aquatic species, but often polluted in areas of 
high frequency. Pressures on Lake Iro's phytocenosis indicate the vulnerability of this ecosystem.

Keywords: Macrophytes, Floristic Diversity, Aquatic Groups, Iro Lake

\section{Introduction}

Les milieux aquatiques et leurs ressources naturelles vivantes fournissent environ les deux tiers des biens et services que la biosphère offre à l'humanité (Agropolis, 2007). Cependant, beaucoup de ces milieux sont de plus en plus fragilisés par les changements climatiques et les modifications de l'usage des sols, et leurs ressources aquatiques mondiales en crise en raison de la surexploitation. Au Tchad, les écosystèmes naturels sont marqués par une dégradation généralisée dont les causes sont soit naturelles (déficit ou irrégularité de la pluviométrie ou baisse constante du niveau des eaux de surface), soit anthropiques (forte démographie, instabilité politique, prélèvement illégal...). Le cas des écosystèmes lacustres est encore plus préoccupant (débit de plus en plus faible des affluents, exploitation irrationnelle, surpâturage, présence des espèces invasives etc.). La préservation des ressources en eau et la conservation des écosystèmes aquatiques constituent de ce fait une priorité pour les décennies à venir (Lacroix et Danger, 2008).

Sur le modèle de la norme européenne, le terme " macrophyte » désigne toutes les plantes aquatiques visibles à l'œil nu, dont les plantes vasculaires, les bryophytes et les algues macroscopiques (OFEV, 2009). Ces plantes aquatiques jouent un rôle important à travers les réseaux trophiques, les processus écologiques d'oxygénation et de purification de l'eau, et aussi dans le maintien de l'équilibre des hydrosystèmes (Fazul et al, 2013 ; Weigleb, 1988). En assurant la production de la biomasse, les macrophytes reflètent le niveau et l'état de fonctionnement du système, et sont susceptibles de renseigner sur un dérèglement de l'ensemble des flux de matières et d'énergie caractérisant ce réseau (Chauvin et al, 2014). Malheureusement, la flore et la végétation aquatiques du lac Iro sont moins connues que celles des autres lacs tels que le lac Tchad (Lemoalle et al., 2012 ; Iltis et Lemoalle, 1979 ; Carmouze et al., 1978 ; Léonard, 1974) et le lac Fitri (Bechir et al, 2019 ; Mikail et al., 2018). Or, le lac Iro est le paradis d'une faune aviaire très nombreuse et très diversifiée. Il est aussi réputé pour sa production de poissons fumés qui ravitaille les marchés de Sarh, Moundou, Abéché et Mongo ainsi que ceux des pays voisins comme le Nigéria et la République centrafricaine (Saunier et al., 2017). Le but de cette étude est de fournir des informations nécessaires sur les macrophytes du lac permettant d'élaborer un schéma efficient de gestion rationnelle. 


\section{Matériel et méthodes}

\section{Présentation de la zone d'étude}

Le lac Iro, situé dans la Province du Moyen Chari, a donné son nom au département du Lac Iro, dont le chef-lieu est la ville de Kyabé. Ses coordonnées géographiques centrées sont $10^{\circ} 6^{\prime} 0 " \mathrm{~N}$ et $19^{\circ} 25^{\prime} 0$ " E. Il mesure $13 \mathrm{Km}$ de long et $11 \mathrm{Km}$ de large, sa superficie en eau moyenne est de 200 km2 (Paigbc, 2012), culmine à $386 \mathrm{~m}$ d'altitude et sa profondeur maximale est de $4 \mathrm{~m}$ à l'étiage. Il est alimenté par les eaux des pluies et les eaux de crue du Bahr Salamat via la rivière d'Iro qui lui sert également de déversoir à la crue. Ses fluctuations saisonnières de niveau sont de l'ordre de 2,5 $\mathrm{m}$ avec un minimum en juillet et un maximum en octobre.

Les sols sont hydro-morphes à pseudo-gley. Ils se sont formés sur la série sableuse à sablo-argileuse récente qui représente les bourrelets d'anciens cours d'eau issus autrefois du Bahr Azoum (Pias et Barbery, 1965). Le lac Iro est entouré par une savane arbustive des zones d'inondation (Pias, 1970) caractérisée par l'association des plantes suivantes : Combretum glutinosum, Piliostigma reticulatum, Acacia sieberiana, Ziziphus mauritiana, Randia nilotica, Andira inermis, Mitragyna inermis, Diospyros mespiliformis, Acacia scorpioides var. nilotica, Acacia seyal, Crataeva adansonii, Pseudocedrela kotschyi, Gardenia ternifolia,

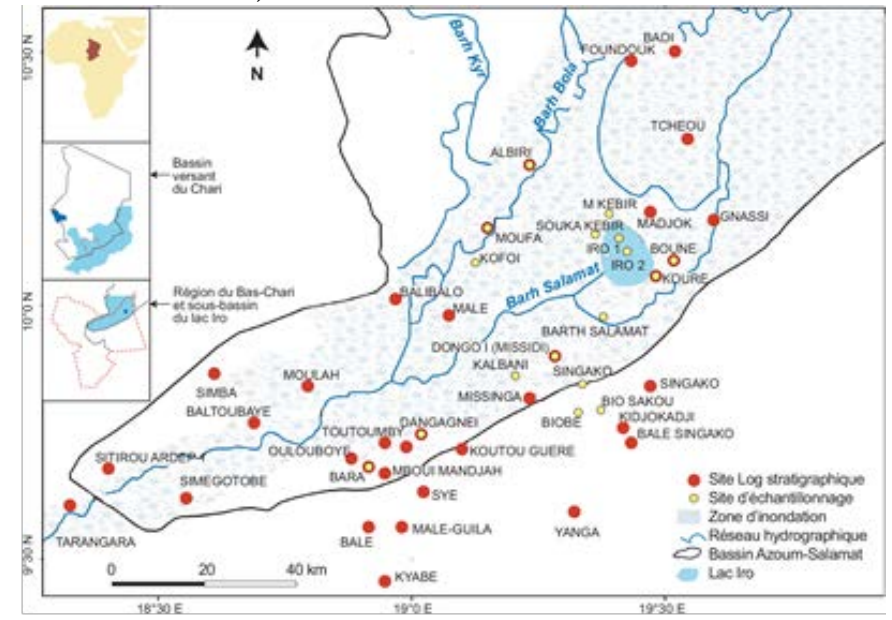

Figure 1. Carte de la localisation du Lac Iro et de ses environs (Amngar et al., 2018)

La méthode utilisée pour cette étude est celle des transects orientés perpendiculairement aux rives et disposés de façon aléatoire. 12 transects ont été réalisés en empruntant les pistes taillées dans la végétation par les pêcheurs installés autour du lac. Les transects permettent d'inventorier et de localiser de façon précise toutes les espèces (patrimoniales, protégées, menacées, rares etc.). Ils permettent également de mesurer des changements d'une communauté à une autre, dus à des gradients environnementaux (humidité, 
topographie, profondeur, etc.), de visualiser la succession de la végétation et d'avoir ainsi une idée de l'influence de certains facteurs écologiques (Jouret, 1972). Cette étude s’est déroulée pendant le mois de mars.

La typologie des groupements végétaux a été réalisée par la méthode phytosociologique (Gillet et al, 1991). Un relevé phytosociologique sur un quadrat de 1 (1x1) à 9 (3x3) m2 est échantillonné le long de chaque transect selon l'homogénéité de la végétation. Le nombre de relevés par transect dépend donc de l'hétérogénéité de la végétation et la longueur des transects est en fonction de la progression de la végétation vers le centre du lac. Le nombre de relevés par transect varie de 12 à 50. Pour chaque relevé, les espèces sont accompagnées des indices d'abondance-dominance (BraunBlanquet, 1932). La fréquence de chaque espèce est calculée et représente le nombre de transects où l'espèce est présente par rapport au nombre total de transects. Certains taxons sont identifiés sur place, d'autres conservés dans des flacons ou mis en herbier pour identification au laboratoire. A cet effet, les flores du Sénégal (Vandhen Berghen, 1998) et du Tchad (César et Chatelain, 2019) ont été mises à contribution. L'approche taxonomique utilisée est basée sur la dernière version APG IV de 2016.

Les descripteurs taxonomiques ont été évalués en utilisant un certain nombre d'indices :

- L'indice de diversité de Shannon est évalué par la formule suvante : $\mathrm{H}^{\prime}=-\Sigma((\mathrm{Ni} / \mathrm{N}) * \log 2(\mathrm{Ni} / \mathrm{N}))$

$\mathrm{Ni}$ : nombre d'individus d'une espèce donnée, i allant de 1 à $\mathrm{S}$ (nombre total d'espèces).

$\mathrm{N}$ : nombre total d'individus.

Il est exprimé en bit et varie généralement entre 1 et 5 bits. Entre 0 et 2,5 il peut être supposé faible (cas des stations spécialisées où l'on note généralement des phénomènes de dominance d'une espèce ou d'un petit nombre d'espèces sur l'ensemble des espèces de la communauté) ; entre 2,6 et 3,9 il peut être supposé moyen et l'indice est maximal (4 à 5) quand tous les individus sont répartis d'une façon égale sur toutes les espèces (Frontier, 1983).

- L’indice d'équitabilité de Piélou aussi appelé indice d'équirépartition (Blondel, 1979) traduit le degré de diversité atteint par rapport au maximum théorique :

$\mathrm{H} / \log (\mathrm{RS})$

$\mathrm{H}$ : l'indice de Shannon.

RS (Richesse spécifique de Pielou) : nombre d'espèces

Il est compris entre 0 et 1 . Il tend vers 0 lorsque la quasi-totalité des individus appartiennent à une seule espèce et prend la valeur 1 lorsque toutes les espèces ont exactement le même recouvrement. 
- L'indice de Simpson mesure la probabilité pour que deux individus tirés au hasard dans un peuplement donné soient de la même espèce (Dajoz, 2006) : $\mathrm{D}=\Sigma \mathrm{Ni}(\mathrm{Ni}-1) / \mathrm{N}(\mathrm{N}-1)$

$\mathrm{Ni}$ : nombre d'individus de l'espèce donnée.

$\mathrm{N}$ : nombre total d'individus.

Cet indice aura une valeur de 0 pour indiquer le maximum de diversité, et une valeur de 1 pour indiquer le minimum de diversité. L'utilisation simultanée de ces trois indices permet une étude plus complète des informations concernant la structure des communautés (Grall et Coïc, 2005).

\section{Traitement des données}

Le logiciel PAST (PAlaeontological STatistics) 4.03 est utilisé pour les analyses statistiques et pour calculer les indices de diversité. C'est un logiciel de statistiques pour les sciences de la nature qui regroupe en un seul logiciel, des fonctions de tests statistiques classiques et de visualisation de données qu'on ne trouve en général que dans des logiciels spécialisés (XLSTAT, canoco, ....). Pour cette étude, il a permis d'effectuer la DCA (Detrented Correspondance Analyse) qui est une version améliorée de l'AFC (Hill et Gauch, 1980) pour individualiser les groupements végétaux ; de visualiser les similitudes entre les transects ; de calculer les indices de Shannon Weaver, de Simpson, d'équitabilité de Piélou, etc.

Le traitement d'une image satellitaire a permis d'établir une carte de végétation du lac Iro.

\section{Mesure des paramètres physico-chimiques}

Des appareils de mesure des paramètres environnementaux ont été utilisés au cours de cette étude : Un disque de Secchi muni d'une corde graduée a été utilisé pour la mesure de la transparence de l'eau et la profondeur du lac au niveau de chaque station, la bouteille de Vandom modifiée et munie d'une corde graduée a été utilisée pour le prélèvement d'échantillon d'eau au fond des lacs, pour la mesure des paramètres physico-chimiques en profondeur et un appareil Multimètre de marque WTW Multi 350i v2.20 a été utilisé pour la mesure in situ des paramètres physico-chimique tels que la température de l'eau, la salinité, la conductivité, l'oxygène dissous et les Solides Totaux Dissouts (TDS).

\section{Résultats}

\section{Paramètres physico-chimiques de l'eau du lac}

Les résultats des données sur les paramètres environnementaux du lac Iro en $201 \neg 6$ sont résumés sur le tableau 1. 


\begin{tabular}{|c|c|c|c|c|c|c|c|c|c|}
\hline $\begin{array}{c}\text { Stations } \\
\text { Des pêcheurs }\end{array}$ & Stations & $\begin{array}{c}\mathrm{T} \\
\left({ }^{\circ} \mathrm{C}\right)\end{array}$ & $\mathrm{pH}$ & $\begin{array}{c}\text { Prof. } \\
(\mathrm{m})\end{array}$ & $\begin{array}{c}\text { Turb } \\
(\mathrm{cm})\end{array}$ & $\begin{array}{c}\mathrm{O}_{2} \\
\text { dissous } \\
(\mathrm{mg} / \mathrm{L})\end{array}$ & $\begin{array}{c}\mathrm{Tx} \\
\mathrm{O}_{2} \\
(\%)\end{array}$ & $\begin{array}{c}\text { Cond } \\
(\mu \mathrm{s} / \mathrm{cm})\end{array}$ & $\begin{array}{c}\mathrm{TDS} \\
(\mathrm{mg} / \mathrm{L})\end{array}$ \\
\hline Hadjer & Transect 1 & 32,6 & 7,52 & 0,8 & 15 & 0,02 & 0,4 & 167 & 167 \\
\hline Ngato & Transect 2 & 30,9 & 6,74 & 0,9 & 10 & 0,02 & 0,3 & 171 & 175 \\
\hline Garbahr & Transect 3 & 31,4 & 7,1 & 0,57 & 10 & 0,45 & 0,3 & 164 & 163 \\
\hline Embouchure & Transect 4 & 28,7 & 7,4 & 0,8 & 10 & 0,6 & 8,5 & 166,8 & 167 \\
\hline Charouba & Transect 5 & 30,6 & 7,8 & 0,34 & 10 & 0,46 & 6,4 & 171,4 & 171 \\
\hline Loungou & Transect 6 & 29,9 & 8,3 & 0,5 & 10 & 0,82 & 12,5 & 164,7 & 165 \\
\hline Bouné & Transect 7 & 26,9 & 6,55 & 0,7 & 15 & 0,41 & 4,9 & 244 & 244 \\
\hline Kouré & Transect 8 & 27,8 & 7,18 & 0,8 & 10 & 0,49 & 6,5 & 175,4 & 175 \\
\hline Darbahr & Transect 9 & 29,3 & 8,08 & 0,45 & 12 & 0,54 & 14,3 & 169 & 169 \\
\hline Tiondi & $\begin{array}{c}\text { Transect } \\
10\end{array}$ & 30,4 & 7,72 & 0,25 & 10 & 0,64 & 9 & 168,9 & 169 \\
\hline Wah & $\begin{array}{c}\text { Transect } \\
11\end{array}$ & $\mathbf{2 9 , 6}$ & 7,01 & 0,45 & 11 & 0,14 & 2,1 & 240 & 238 \\
\hline Boum kébir & $\begin{array}{c}\text { Transect } \\
12\end{array}$ & $\mathbf{2 9 , 5}$ & $\mathbf{7 , 1}$ & 0,3 & 10 & 0,1 & 1 & 275 & 276 \\
\hline Moyennes & & $\mathbf{2 9 , 8}$ & $\mathbf{7 , 3 8}$ & $\mathbf{0 , 5 7}$ & $\mathbf{1 1 , 0 8}$ & $\mathbf{0 , 3 9}$ & $\mathbf{5 , 4 3}$ & $\mathbf{1 1 9 , 1 7}$ & $\mathbf{1 8 9 , 9 2}$ \\
\hline
\end{tabular}

Tableau 1. Résultats des données physico-chimiques de l'eau.

Prof. : Profondeur ; Turb : Turbidité ; Cond : Conductivité ; TDS : Solides Totaux Dissouts.

La température moyenne globale du lac lors de cette étude a été de $29,8^{\circ}$ C. La température moyenne la plus basse $\left(26,9^{\circ} \mathrm{C}\right)$ a été enregistrée sur le transect 7 et la température moyenne la plus haute $\left(32,6^{\circ} \mathrm{C}\right)$ a été observée au niveau du transect 1 . Le $\mathrm{pH}$ moyen du transect 9 est le plus élevé $(8,3)$ et la moyenne minimale $(6,55)$ est rencontrée sur le transect 7 . La profondeur maximale de $0,9 \mathrm{~m}$ a été rencontrée sur le transect 2 . Les valeurs moyennes de la transparence ont fluctué entre $10 \mathrm{~cm}$ et $15 \mathrm{~cm}$ dans la plupart des transects. La valeur moyenne de l'oxygène dissous a été plus forte $(0,82 \mathrm{mg} / \mathrm{L})$ au niveau du transect 6 et faible $(0,02 \mathrm{mg} / \mathrm{L})$ au niveau des transects 1 et 2 . Le taux moyen de saturation en oxygène dissous le plus élevé (14,3\%) a été enregistré au niveau du transect 9 et le plus bas $(0,3 \%)$ au niveau des transects 2 et 3 . Le transect 12 a été marqué par une conductivité moyenne élevée $(275 \mu \mathrm{s} / \mathrm{cm})$ et par un TDS également plus important (276 mg/l), au transect 3 la conductivité (164 $\mu \mathrm{s} / \mathrm{cm})$ et le TDS minimal (163 mg/l) ont été les moins importants. Ces résultats montrent une relative homogénéité du milieu sauf au niveau de l'oxygène dissous et du taux d'oxygène où les amplitudes entre certains transects sont assez considérables.

La figure 2 visualise l'analyse canonique des correspondances de 12 transects, 30 espèces végétales recensées et 8 variables physico-chimiques de l'eau. Elle a permis de mettre en évidence la répartition des transects en fonction des variables du milieu. L'axe factoriel F1 discrimine les transects 1 , 2, 3, 5, 7 et 10 qui sont négativement corrélés à la température, à la profondeur 
et à la turbidité. Les autres transects positivement corrélés par l'axe F1 sont séparés en deux groupes suivant l'axe F2. Du côté négatif de l'axe F2, les transects 8,9 et 12 sont regroupés autour du $\mathrm{pH}$ tandis que du côté positif de cet axe, s'individualisent les transects 4, 6 et 11 inféodés de l'oxygène dissous, du taux d'oxygène et les solides totaux dissouts.

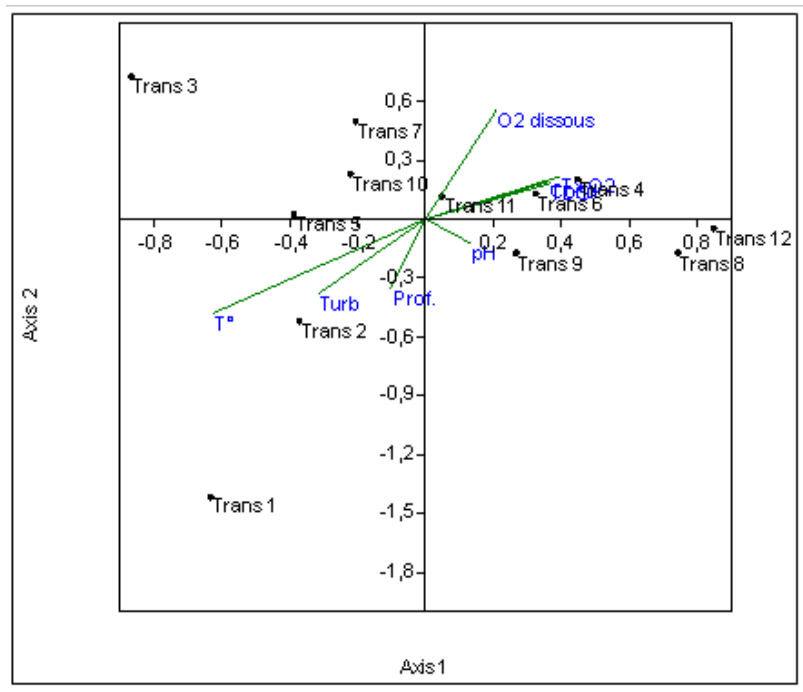

Figure 2.Analyse canonique des correspondances des paramètres physico-chimiques et des transects.

\section{Diversité floristique}

Les valeurs des indices de Shannon, de Simpson et d'équitabilité de Piélou enregistrées sur les différents transects sont matérialisées sur le tableau 2 et la figure 3.

\begin{tabular}{|c|c|c|c|c|c|c|c|c|c|c|c|c|}
\hline Transects & 1 & 2 & 3 & 4 & 5 & 6 & 7 & 8 & 9 & 10 & 11 & 12 \\
\hline Taxa_S & 12 & 10 & 13 & 11 & 11 & 11 & 9 & 10 & 7 & 8 & 11 & 11 \\
\hline Dominance_D & 0,17 & 0,16 & 0,13 & 0,14 & 0,11 & 0,12 & 0,2 & 0,12 & 0,16 & 0,178 & 0,129 & 0,154 \\
\hline Simpson_1-D & 0,83 & 0,84 & 0,87 & 0,86 & 0,89 & 0,88 & 0,8 & 0,88 & 0,84 & 0,822 & 0,871 & 0,846 \\
\hline Shannon_H & 1,98 & 1,99 & 2,24 & 2,12 & 2,29 & 2,22 & 1,86 & 2,23 & 1,88 & 1,889 & 2,212 & 2,089 \\
\hline Equitability_J & 0,8 & 0,86 & 0,87 & 0,88 & 0,95 & 0,92 & 0,84 & 0,97 & 0,97 & 0,909 & 0,922 & 0,871 \\
\hline
\end{tabular}

Tableau 2. Variation des indices de Shannon, de Simpson et d'Equitabilité

Le transect le plus long est constitué de 13 espèces tandis le plus court n'en compte que 7. Les valeurs de l'indice de Shannon (H') qui varient de 1,88 à 2,29 bits, ont révélé que les transects 9 et 10 sont les moins diversifiés alors les transects 5 et 3 sont les plus diversifiés. La Figure 3 montre que les indices de Simpso 


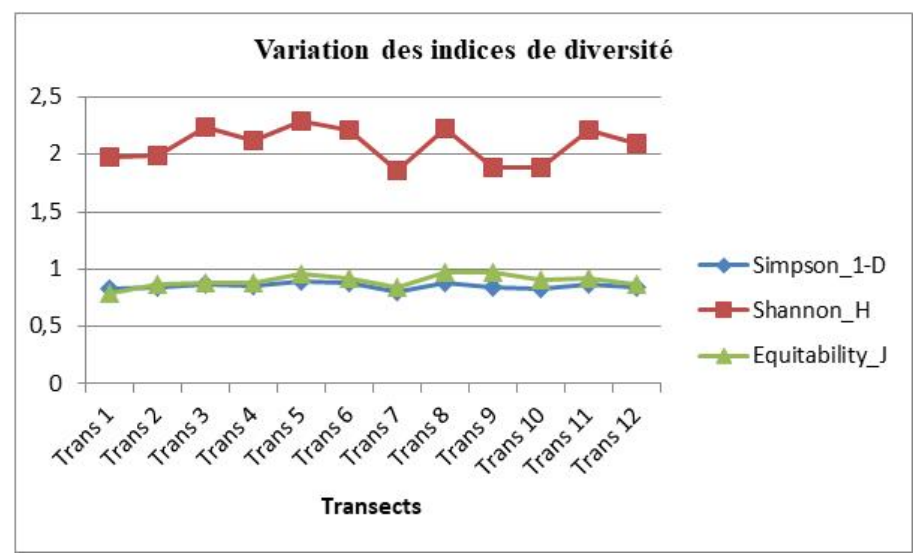

Figure 3.Courbes des variations des indices de Shannon, de Simpson et d'Equitabilité sur les différents transects.

\section{Flore}

Il a été recensé 30 espèces reparties entre 21 genres et 12 familles. Cette flore est composée de 96,42\% d'Angiospermes et de 3,58\% de fougères. La famille des Poaceae est la mieux représentée (20\%) suivie des Fabaceae (18\%) et des Cyperaceae (11\%) (Figure 4). Les espèces les plus fréquentes et présentes sur presque tous les transects sont: Echinochloa colona, Echinochloa stagnina, Neptunia oleracea, Ceratophyllum demersum, Pistia stratioites, etc.

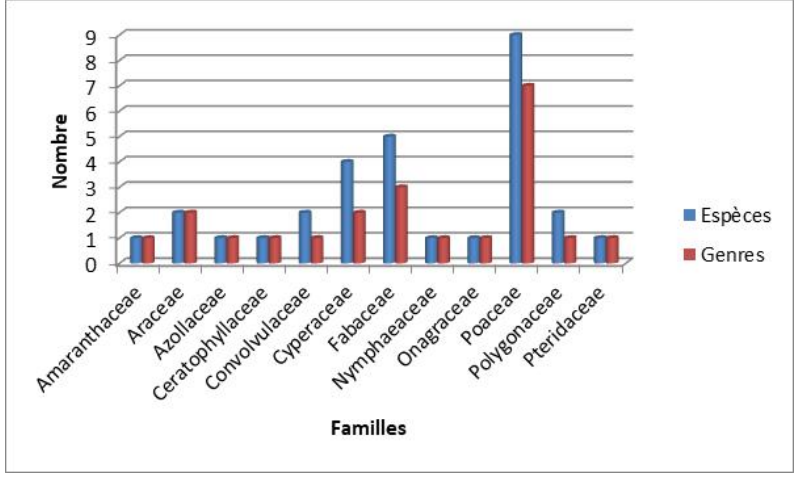

Figure 4.Spectre des Familles, Genres et Espèces dans le lac Iro

Tableau 3. Liste des macrophytes rencontrées dans le lac Iro n et d’Equitabilité de Piélou ont décrit les mêmes allures.

\begin{tabular}{|c|c|c|c|c|}
\hline Espèces & Familles & Type biologique & Fr (\%) & Codes \\
\hline $\begin{array}{c}\text { Aeschynomene elaphroxylon } \\
\text { (Guill \& Perr.) Taub }\end{array}$ & Fabaceae & Hélophyte & 25 & Ael \\
\hline $\begin{array}{c}\text { Aeschynomene afraspera J. } \\
\text { Léonard. }\end{array}$ & Fabaceae & Hélophyte & 25 & Aaf \\
\hline Aeschynomene indica L. & Fabaceae & Hélophyte & 8 & Ain \\
\hline
\end{tabular}




\begin{tabular}{|c|c|c|c|c|}
\hline $\begin{array}{c}\text { Anosporum pectinatus (Vahl) } \\
\text { Lye }\end{array}$ & Cyperaceae & Hélophyte & 8 & Cpe \\
\hline $\begin{array}{c}\text { Azolla pinnata subsp africana } \\
\text { Desv. }\end{array}$ & Azollaceae & Hydrophyte flottante libre & 50 & Api \\
\hline $\begin{array}{c}\text { Centrostachys aquatica (R. } \\
\text { Br) Wall. }\end{array}$ & Amaranthaceae & Hélophyte & 8 & Caq \\
\hline Ceratophyllum demersum L. & Ceratophyllaceae & $\begin{array}{l}\text { Hydrophyte flottante } \\
\text { Immergée libre }\end{array}$ & 100 & Cde \\
\hline $\begin{array}{l}\text { Ceratopteris thalictroides } \\
\text { (L.) Brongn. }\end{array}$ & Pteridaceae & $\begin{array}{l}\text { Hydrophyte flottante } \\
\text { émergée }\end{array}$ & 8 & Cth \\
\hline Cyperus laevigatus L. & Cyperaceae & Hélophyte & 8 & Cle \\
\hline Cyperus pustulatus R. Br. & Cyperaceae & Hélophyte & 100 & $\mathrm{Cpu}$ \\
\hline Echinochloa colona (L) Link & Poaceae & $\begin{array}{l}\text { Hydrophyte flottante } \\
\text { Immergée libre }\end{array}$ & 100 & Eco \\
\hline $\begin{array}{l}\text { Echinochloa stagnina (Retz) } \\
\text { P. Beauv. }\end{array}$ & Poaceae & $\begin{array}{l}\text { Hydrophyte flottante } \\
\text { Immergée libre }\end{array}$ & 83 & Est \\
\hline $\begin{array}{c}\text { Eragrostis atrovirens (Desf) } \\
\text { Trin. Ex Steud. }\end{array}$ & Poaceae & Hélophyte & 33 & Eat \\
\hline Ipomoea aquatica Forssk. & Convovulaceae & Liane Hélophyte & 17 & Iaq \\
\hline Ipomoea rubens Choisy & Convovulaceae & Liane Hélophyte & 8 & Iru \\
\hline Lemna minor L. & Araceae & $\begin{array}{l}\text { Hydrophyte flottante } \\
\text { Immergée }\end{array}$ & 83 & Lmi \\
\hline Leersia hexandra Sw. & Poaceae & Hélophyte & 83 & Lhe \\
\hline $\begin{array}{l}\text { Ludwigia adscendens subsp } \\
\text { diffusa (Forssk) P.H. Raven }\end{array}$ & Onagraceae & $\begin{array}{l}\text { Hydrophyte flottante } \\
\text { Fixée } \\
\end{array}$ & 8 & Lst \\
\hline Neptunia oleracea Lour. & Fabaceae & Hélophyte & 8 & Nol \\
\hline Nymphaea lotus L. & Nymphaeaceae & $\begin{array}{l}\text { Hydrophyte flottante } \\
\text { Fixée }\end{array}$ & 75 & Nlo \\
\hline $\begin{array}{c}\text { Persicaria decipiens (R. Br) } \\
\text { K. L. Wilson } \\
\end{array}$ & Polygonaceae & Hélophyte & 17 & Pde \\
\hline $\begin{array}{c}\text { Persicaria lapathifolia (L.) } \\
\text { Delarbre } \\
\end{array}$ & Polygonaceae & Hélophyte & 17 & Pla \\
\hline $\begin{array}{c}\text { Phragmites australis (Cav.) } \\
\text { Trin. Ex Steud. }\end{array}$ & Poaceae & Hélophyte & 8 & Pau \\
\hline Pistia stratiotes L. & Araceae & $\begin{array}{l}\text { Hydrophyte flottante } \\
\text { libre }\end{array}$ & 17 & Pst \\
\hline Pycreus mundtii Ness & Cyperaceae & Hélophyte & 8 & Pmu \\
\hline Sesbania sesban (L) Merril & Fabaceae & Hélophyte & 17 & Sse \\
\hline $\begin{array}{l}\text { Setaria geminata (Forssk.) } \\
\text { Veldkamp }\end{array}$ & Poaceae & Hélophyte & 8 & Pge \\
\hline Sporobolus robustus Khunt & Poaceae & Hélophyte & 17 & Sro \\
\hline Sporobolus spicatus Khunt & Poaceae & Hélophyte & 8 & Ssp \\
\hline $\begin{array}{l}\text { Vossia cuspidata (Roxb) } \\
\text { Griff }\end{array}$ & Poaceae & $\begin{array}{l}\text { Hydrophyte flottante } \\
\text { Immergée libre }\end{array}$ & 33 & Vcu \\
\hline
\end{tabular}

Tableau 3. Liste des macrophytes rencontrées dans le lac Iro n et d’Equitabilité de Piélou ont décrit les mêmes allures. 
Le tableau 3 dresse la liste des espèces présentes dans le lac Iro, les types biologiques ainsi que les codes utilisés dans les analyses statistiques. La définition des types biologiques fait référence à Montegut (1987) :

- Les hélophytes : ce sont des plantes amphibies dont la base est normalement dans l'eau et dont le développement des parties reproductrices nécessite le contact avec l'atmosphère

- Les hydrophytes fixés à feuilles flottantes affleurantes possèdent un appareil radiculaire, leurs parties végétatives traversent la colonne d'eau par les tiges ou les pétioles et viennent s'étaler à la surface de l'eau (feuilles).

- Les hydrophytes libres ou pleustophytes ne possèdent aucun système de fixation sur le substrat, ils peuvent être soit flottants, soit nageants en pleine eau comme Utricularia sp.

\section{Groupements végétaux aquatiques}

L'analyse DCA de la végétation a permis de distinguer 7 groupements végétaux suivant les recouvrements des espèces majeures.

Trois types des groupements (G1, G2 et G3) sont identifiés sur tous les transects. La physionomie floristique de ces trois groupements marque la zonation globale du lac. La figure 5 rassemble les diagrammes des transects $(1,5,6,7,8$ et 9$)$ qui ne renferment principalement que ces trois groupements accompagnés de faibles variations de composition floristique suivant les milieux.

- Groupement à Echinochloa stagnina (G1).

Ce groupement est essentiellement représenté par Echinochloa stagnina en peuplement souvent très dense (recouvrement de 100\%) et monospécifique. On note la présence plus ou moins discrète de Ceratophyllum demersum, Aeschynomene crassicaulis, Azolla pinnata subsp africana et Lemna minor. Ce groupement flottant peut parfois dépasser 1,75 $\mathrm{m}$ de hauteur et colonise régulièrement l'auréole interne la plus profonde $(>0,30 \mathrm{~m})$ des transects sur une bande qui s'étale de 50 à $500 \mathrm{~m}$.

- Groupement Echinochloa colona (G2).

Dans ce groupement, Echinochloa colona a une position dominante comme Echinochloa stagnina dans le Précédent. Ce groupement occupe la ceinture périphérique du lac et souvent dégradée par le surpâturage et les zones peu profondes $(0$ à $0,30 \mathrm{~m})$. La hauteur de cette végétation atteint rarement 1 $\mathrm{m}$ et souvent dégradée par le surpâturage. Dans les parties où Echinochloa colona est moins dense, on rencontre invariablement des espèces enracinées dans la vase ou flottantes telles que : Neptunia oleracea, Pistia stratioites, Ceratophyllum demersum, Persicaria decipiens, Ipomoea aquatica...

- Groupement Neptunia oleracea et Ludwigia adscendens subsp. diffusa (G3)

La végétation de ce groupement se trouve également sur presque tous les transects. C'est un ensemble de tapis flottants de 10 à $30 \mathrm{~cm}$ de haut de 
composition floristique très variable intercalé entre les deux groupements précédents. Ces deux espèces sont souvent entremêlées avec d'autres espèces rampantes telles que Ipomoea aquatica et Ipomoea rubens dont l'importance varie suivant les transects. On y trouve régulièrement éparpillées dans cette masse : Pistia stratioites, Ceratophyllum demersum, Azolla pinnata subsp africana et Lemna minor.

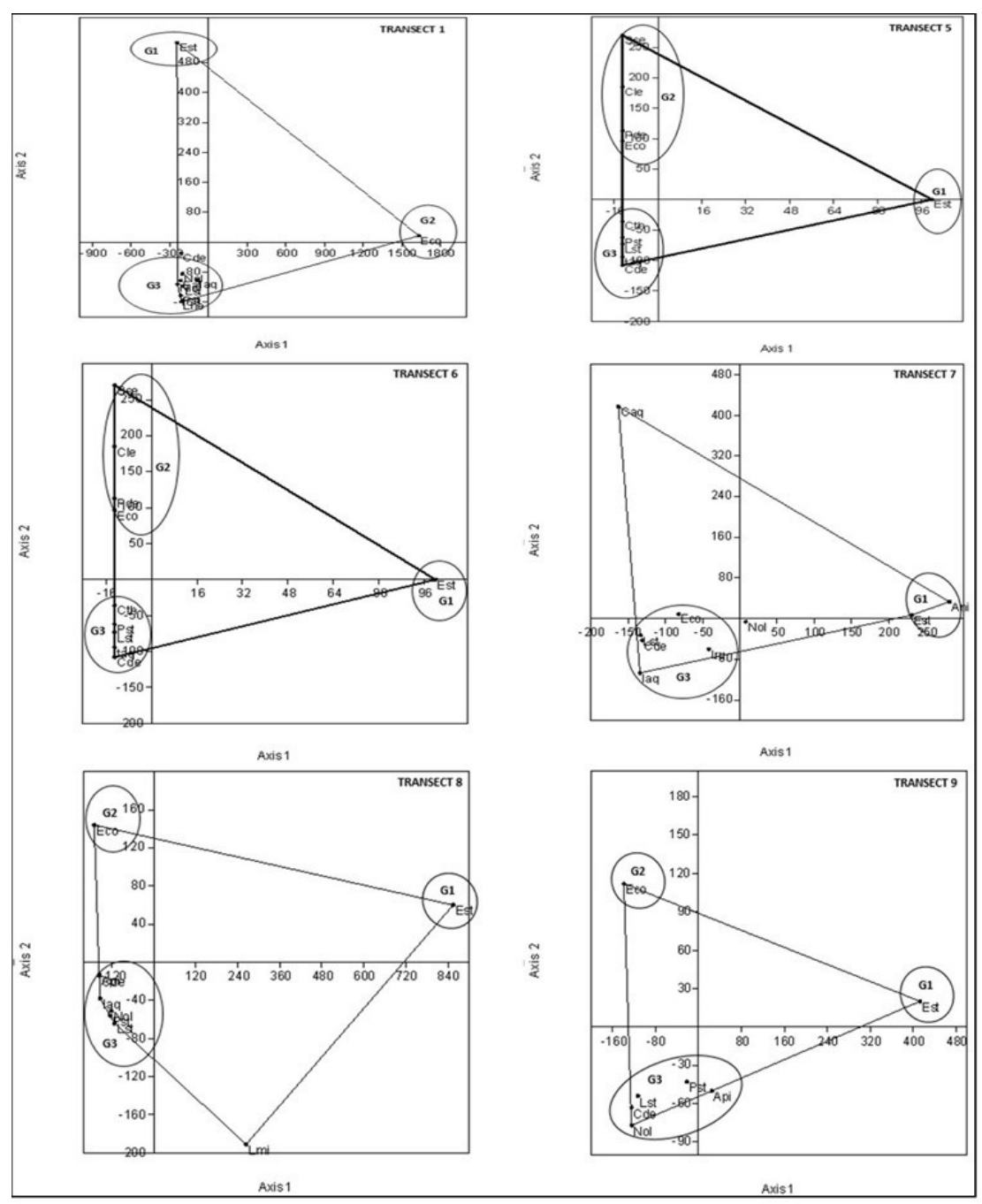

Figure 5.Diagrammes des transects renfermant uniquement les trois principaux groupements à Echinochloa stagnina (G1), Echinochloa colona (G1) et Neptunia oleracea et Ludwigia adscendens subsp. diffusa (G3)

- Groupement à Phragmites australis (G4) :

Ce groupement est situé sur les berges au point de départ du transect 3 . La principale espèce est le Phragmites australis (figure 6). C'est un 
peuplement d'une hauteur moyenne de $1,80 \mathrm{~m}$. Les autres espèces de ce groupement sont : Persicaria decipiens et Persicaria lapathifolia.

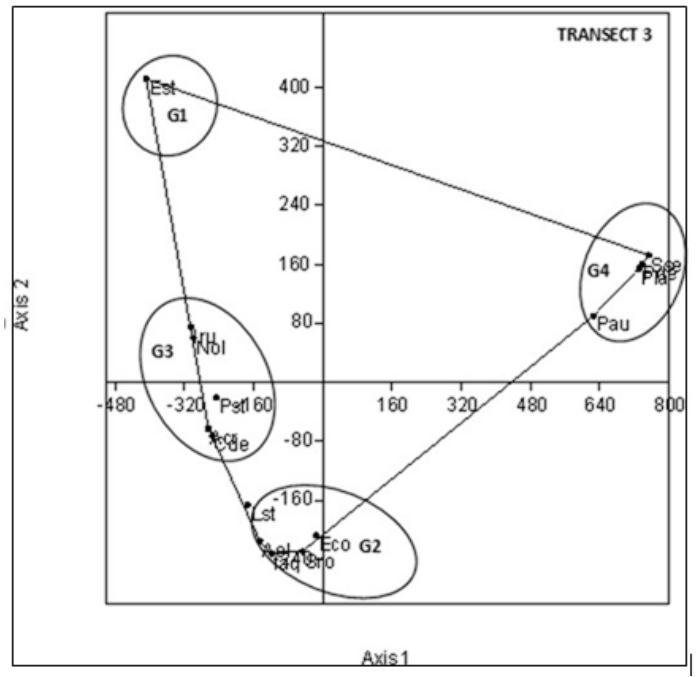

Figure 6 : Diagramme comportant les trois principaux groupements et le groupement à Phragmites australis (G4)

- Groupement à Vossia cuspidata (G5) :

Ce groupement est moins fréquent et ne se rencontre que sur les transects 4 et 10 (Figure 7). L'espèce dominante est le Vossia cuspidata.

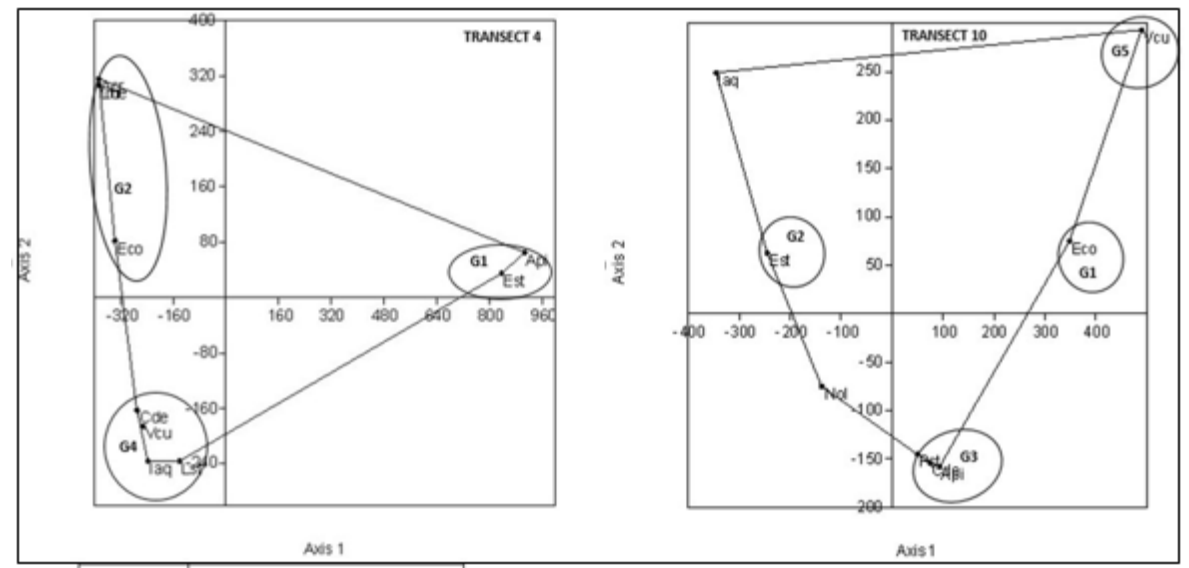

Figure 7 : Diagramme comportant les trois principaux groupements et le groupement à Vossia cuspidata (G5).

- Groupement à Leersia hexandra et Cyperus ssp (G6) :

Sur la figure 8 sont regroupés des transects qui diffèrent des précédents par la présence de certains végétaux amphibies qui deviennent plus ou moins abondants sur les parties plus exondées. Ce sont des fragments de prairies plus 
ou moins sèches constitués de Poaceae telles que Leersia hexandra, Sporobolus spicatus, Sporobolus robustus... et des Cyperaceae telles que Cyperus pustulatus, Anosporum pectinatus et Pycreus mundtii.

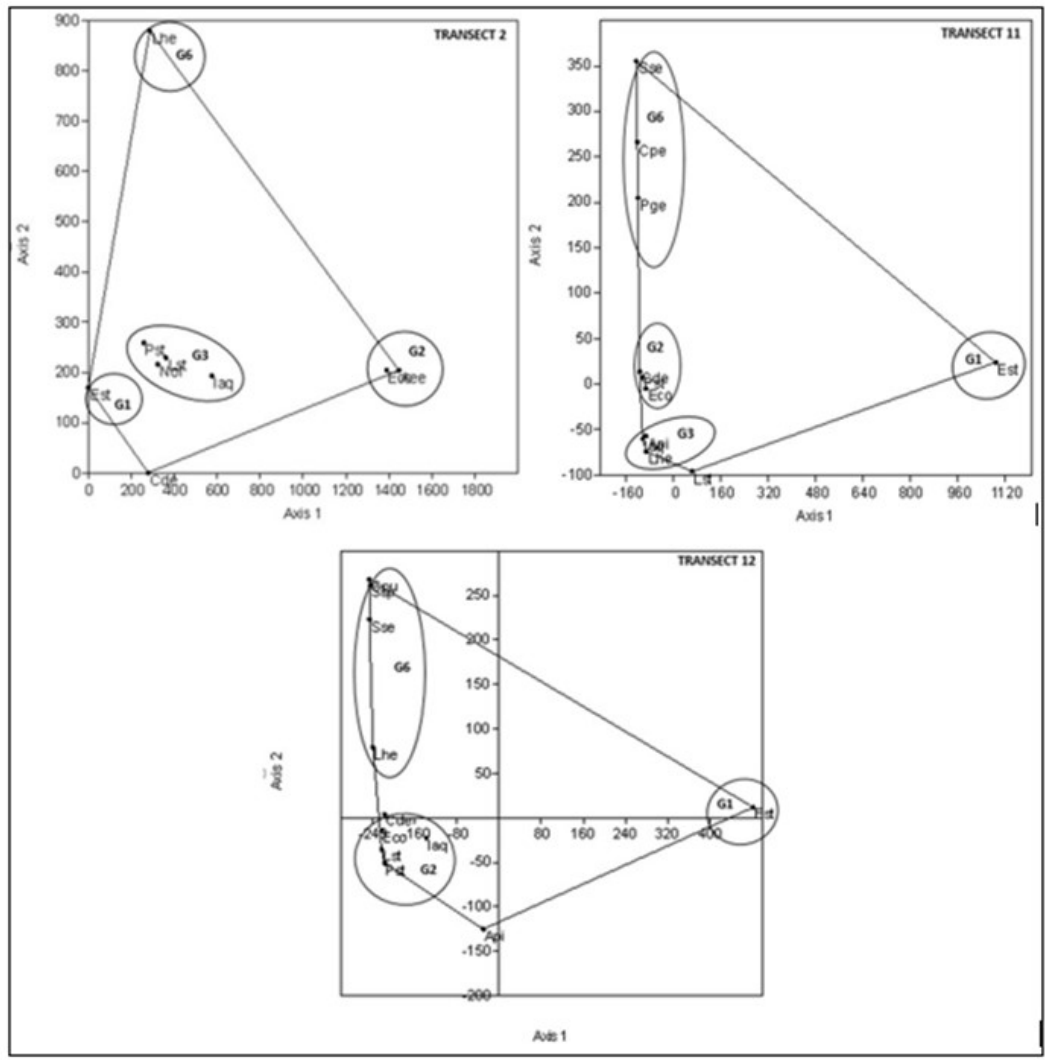

Figure 8 : Diagramme comportant les trois principaux groupements et le groupement à Leersia hexandra et Cyperus ssp (G6).

L'image satellitaire de la figure 9 ci-dessous montre les zonations majeures de la végétation du lac Iro caractérisée par les trois groupements végétaux dominants. 


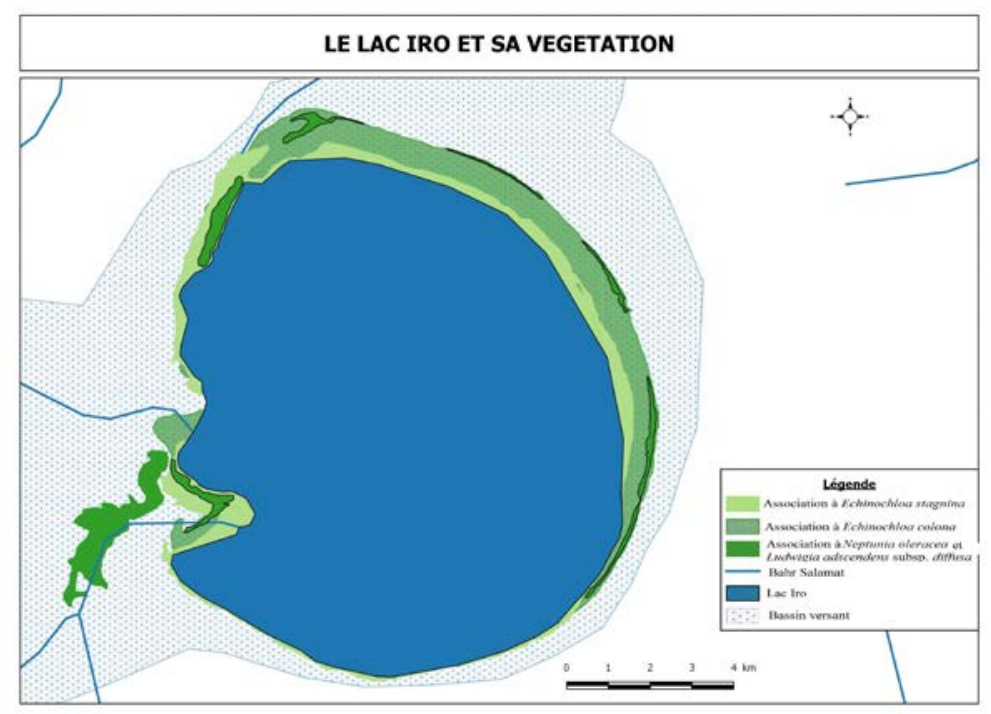

Figure 9. carte de zonation végétale du lac Iro

\section{Discussion}

\section{Caractéristiques physico-chimiques de l'eau du lac}

La température moyenne de 29,8 oC exprime le fait que l'eau du lac est restée assez chaude pendant cette période de l'année entre mars-avril. Elle est comparable à celle de certains cours d'eau au Cameroun (Taffouo et al., 2017). La différence de $5{ }^{\circ} \mathrm{C}$ observée entre le transect le plus chaud et le transect le plus froid pourrait s'expliquer par la densité de la couverture végétale, la profondeur et la turbidité comme ressortie dans l'analyse canonique. De façon globale, la température du lac Iro est favorable aux espèces aquatiques (Noumon et al., 2015 ; Dimon et al., 2014). La valeur moyenne du $\mathrm{pH}(7,38)$ indique une eau proche de la neutralité et de bonne qualité selon la grille de la valeur indicatrice (Beaux, 1998). Si les pH faibles (eaux acides) augmentent notamment le risque de présence de métaux sous une forme ionique plus toxique, ceux qui sont élevés augmentent les concentrations d'ammoniac toxique pour les poissons (De Villier et al., 2005). Cette qualité de l'eau serait due à la présence des macrophytes en décomposition qui baisserait le pH (Atanle et al, 2012). La turbidité est une grandeur qui permet d'évaluer la quantité de la matière en suspension dans l'eau (Houma et al., 2004). La moyenne de cette turbidité est relativement élevée. Ceci pourrait s'expliquer par la présence massive de la matière organique végétale en décomposition ou issue des déjections des animaux. L'oxygène dissous présente une valeur moyenne très faible inférieure à $1 \mathrm{mg}$ d'O2 par litre et indique un état proche de l'anaérobie (De Villier et al., 2005). Selon ces auteurs, cet état se produit lorsque les processus d'oxydation des déchets minéraux, de la matière organique et des nutriments consomment plus 
d'oxygène que celui disponible. Cela confirme l'état de la pollution du milieu par les activités humaines, les déchets animaux et la matière organique en décomposition. La conductivité d'une eau est une expression numérique de sa capacité à conduire le courant électrique. La conductivité d'une eau naturelle est comprise entre 50 et $1500 \mu \mathrm{S} / \mathrm{cm}$. La valeur moyenne de $119,17 \mu \mathrm{s} / \mathrm{cm}$ inférieure à la moyenne standard corrobore une fois de plus, une faible minéralisation du lac Iro et sa grande quantité de la matière organique. Cette situation concorde aussi avec la faible valeur moyenne $(189,92 \mathrm{mg} / \mathrm{L})$ des Solides Totaux Dissous (TDS).

\section{Richesse floristique}

La flore du lac est constituée de 30 espèces reparties entre 21 genres et 12 familles. Elle est essentiellement composée d'Angiospermes et de quelques fougères. Les familles des Poaceae, des Fabaceae et des Cyperaceae sont les mieux représentées. Les valeurs de l'indice de diversité de Shannon qui varient de 1,88 à 2,29 bits montrent que la végétation du lac Iro n'est pas très diversité pendant la période de cette étude. En effet, l'étude a été menée à une période correspondant à la saison de grande décrue du lac. Les espèces inventoriées sont situées à la limite de l'eau car au fur et à mesure que l'eau se retire la zone émergée est soit progressivement occupée par des champs de sorgho ou par les cultures maraichères, soit la végétation systématiquement broutée par le bétail. Les espèces rencontrées sont très majoritairement aquatiques, très peu d'espèces amphibies ou semi-aquatiques des berges. La rareté des Nymphaeaceae et Polygonaceae pendant cette période alors qu'elles sont très répandues dans les eaux douces (Iltis et Lemoalle 1979 ; Saradoum et al, 2012) peut s'expliquer par la forte baisse du niveau de l'eau. Certes la diversité floristique est faible mais les espèces clés de cette végétation telles que Echinochloa colona, Echinochloa stagnina, Voscia cuspidata, Neptunia oleracea et Ludwigia adscendens subsp. diffusa ont des recouvrements très importants formant souvent des peuplements denses. Une espèce de Ptéridophytes, Ceratopteris cornuta, aperçue sur un seul transect et selon les pêcheurs, c'est la première fois qu'elle a fait son apparition en 2015 sur le lac. Elle n’a pas été revue en 2016.

\section{Groupements végétaux}

Les résultats de cette étude ont fait ressortir plusieurs groupements déjà décrits et validés. De ce fait, les désignations syntaxonomiques correspondant à leurs éléments caractéristiques sont utilisées en remplacement du mot groupement.

L'association à Echinochloa stagnina peut être assimilé à la Echinochletum stagninae décrit dans l'ex Congo Belge actuellement République Démocratique du Congo (Robyns, 1936). Il occupe la ceinture 
intérieure du lac formant de vastes îles flottantes presque pures. On y observe parfois quelques incursions de Ceratophyllum demersum, Aeschynomene crassicaulis, Azolla pinnata subsp africana et Lemna minor à la périphérie. En Afrique tropicale, on trouve Echinochloa stagnina du niveau de la mer jusqu'à 2300 m d'altitude, en eaux peu profondes, dans les marécages et sur des sols argileux régulièrement inondés (Brink, 2006). Cette espèce vulgairement appelée bourgou ou roseau sucré a une haute potentialité fourragère mais joue également un rôle épurateur des eaux polluées (Moustapha et al., 2012).

Le Echinochloetum colonae est décrit en combinaison avec d'autres espèces dans une nouvelle alliance Panico laeti-Echinochloion colonae constitué de 5 associations dans les zones semi-arides et subhumides de l'Afrique de l'ouest (Müller et Wezel, 2006). Il occupe la ceinture périphérique du lac et les espèces qui accompagnent ce groupement sont majoritairement des hydrophytes fixées ou hélophytes des genres Sesbania, Aeschynomene, Sporobolus et Cyperus. Echinochloa colona est considérée par certains auteurs (Andrew, 2018) comme une espèce invasive qui a un impact négatif sur la biodiversité des écosystèmes aquatiques ou semiaquatiques. Cette partie de la végétation subit toute sorte de pressions (surpâturage, piétinement et feux de brousse).

L'association à Neptunia oleracea et Ludwigia adscendens subsp. diffusa peut être classée la classe des Potametea Tüxen et Preising 1942. Elle est constituée d'une mosaïque d'espèces telles que Pistia stratioites, Ceratophyllum demersum, Ipomoea aquatica et Ipomoea rubens qui entrent dans la dénomination de plusieurs associations végétales selon leur importance. Par exemple un groupement à Pistia stratioites a été déterminé dans le Ferlo (Sarr et al., 2001). En Afrique, la FAO (2002) a qualifié Pistia stratioites d'espèce envahissante contre laquelle il faut lutter. Un groupement à Azolla pinnata et Ludwigia stolonifera a été décrit dans le delta du fleuve Sénégal et le lac des Guiers (Thiam, 2012). Dans les communautés végétales aquatiques de la classe des Potametea du lac Tonga, Ceratophyllum demersum entre dans la dénomination des certaines Ordres, Alliances ou Associations en compagnie d'autres espèces (Kadid et al., 2007). Cependant, il faut noter que cette espèce présente des difficultés taxonomiques. Ceratophyllum demersum est largement utilisé en Afrique tropicale (Ndour, 2019 ; Potères, 1950) alors que certains auteurs (Vandhen Berghen, 1998) ont signalé que la famille de Cerathophyllaceae n'est représentée en Afrique occidentale que par une espèce qui est Ceratophyllum muricatum et cela est confirmé par la répartition de Ceratophyllum demersum sur d'autres continents (Syed et al., 2018 ; Les, 1986).

L'association à Phragmites australis peut s'intégrée dans le Phragmitetum australis (Lebrun 1947) très répandue à travers le monde où plusieurs études ont démontré qu’il est envahissant (Fetnaci et al., 2019 ; 
Lavoie, 2012). Autour du lac Iro, elle a une extension limitée au départ d'un seul transect sur quelque vingtaine de mètre. Cela peut s'expliquer par le fait que ce groupement soit constamment soumis aux feux de brousse.

L'association à Vossia cuspidata ou le Vossietum cuspidatae déjà signalée en République Démocratique du Congo (Lebrun 1947 ; Robyns, 1936), présente les mêmes caractéristiques que le groupement à Echinochloa stagnina et Echinochloa colona. Elle forme des ilots flottant plus réduits.

Le groupement à Leersia hexandra et Cyperus ssp est moins bien structuré que les autres et très fragmentaire. Il occupe les berges temporairement inondées et dégradé par diverses pressions anthropiques.

\section{Menaces et pressions}

La végétation du lac Iro est soumise à des pressions diverses qui menaceraient sa dynamique si des mesures de conservation ne sont pas prises. La forte concentration quasi permanente entraine des conséquences sur les possibilités de régénération et sur la qualité de l'eau. Les déjections animales sont des sources de pollution et de l'eutrophisation de l'eau. Elles influent également sur l'oxygénation de l'eau. Les éleveurs coupent les herbes des parties profondes comme fourrage tandis que les pêcheurs font des trouées importantes dans la végétation pour optimiser les prises de poissons. Ce dégarnissement de la surface de l'eau s'accompagne d'une forte évaporation réduisant rapidement la superficie du lac. En plus, le prélèvement d'espèces d'intérêt socioéconomique risque de favoriser la prolifération des espèces envahissantes qui actuellement n'ont pas un impact significatif. Tous ces facteurs conduiront forcement à un changement de la composition des phytocénoses et de la biodiversité.

\section{Conclusion}

L'étude dans lac Iro a permis d'apprécier la qualité de l'eau, d'évaluer la diversité floristique et d'identifier des groupements végétaux dont certains peuvent être intégrés dans des syntaxons existants. La principale pression qui s'exerce sur cette végétation est le surpâturage mais aussi certaines pratiques de la pêche. Si la tendance à l'augmentation croissante de la quantité de matière en décomposition issue de ces activités et la dégradation du couvert végétal ne s'inversait pas, la capacité épuratrice des macrophytes sera dépassée par l'ampleur de la pollution. On évoluerait vers un état trophique de l'eau néfaste au développement des espèces animales et végétales aquatiques. Cette éventuelle catastrophe peut être évitée si des mesures efficientes de protection et de conservation sont prises et appliquées dès maintenant. Ces mesures doivent s'orienter vers une maîtrise de la prolifération des espèces envahissantes, la salubrité des berges et la promotion des espèces a haute 
potentialité épuratrice. Cette étude doit être suivie par d'autres travaux à des périodes différentes de l’année pour être plus complète.

\section{Remerciements}

Les auteurs adressent leurs sincères remerciements au projet FSP Grands Ecosystèmes Lacustres Tchadiens (GELT) et la Coopération Française pour avoir initié et accompagné cette recherche. Leur reconnaissance va également à l'endroit de tous les collègues pour leur apport multiforme au cours des missions de terrain et à M. Hoinathy Noubatan pour sa contribution à la réalisation de la carte de végétation.

\section{References:}

1. Agropolis International, (2007). Ecosystèmes aquatiques : Ressources et Valorisation. Dossiers numéro 6. 67 p.

2. Amngar, G., Hamit, A. \& Deschamps P. (2018). Caractérisation des paramètres physico-chimiques des eaux souterraines ; implication pour l'évaluation des potentialités des ressources en eau du bassin hydrogéologique de la région du Lac Iro (République du Tchad). International Journal of Biological and Chemical Sciences, Vol 12, No 5, 2446-2463.

3. Andrew, S.M. (2018). Invasive Species Echinochloa colona Reduces Abundance and Diversity of Resident Plant Communities in Tropical Wetland. Open Journal of Ecology, 8, 483-494.

4. APG IV. (2016). An update of the Angiosperms Phylogeny Group classification for the orders et families of flowering plants. Botanical Journal of Linnean Society, 180, 1-20.

5. Atanle, K., Moctar, L., Bawa, Kokou K. \& Gbandi D. B. (2012). La caractérisation physicochimique et diversité phytoplanctonique des eaux du lac de Zowla (Lac Boko), au Togo. J. Appl. BioSci., 64 : 48474857.

6. Beaux, J. F. (1998). L'environnement, Repères pratiques. Nathan, 160p.

7. Bechir, A. B., Mian-Oudanang, K., Alhassine, M. \& Tchoudiba, B. T. (2019). La végétation pastorale du Lac Fitri : état des lieux et dynamique, IRD, 189-202.

8. Blondel, J. (1979). Biogéographie et écologie. Masson, Paris, 173 p.

9. Braun-Blanquet, J. (1932). Plant sociology: the study of plant communities. McGray Hill, New York, 439 p

10. Brink, M. (2006). Echinochloa stagnina (Retz.) P.Beauv. In: Brink, M. \& Belay, G. (Editors). PROTA (Plant Resources of Tropical Africa / Ressources végétales de l'Afrique tropicale), Wageningen, Netherlands. 
11. Carmouze, J.P., Fotius, G. \& Leveque, C. (1978). Influence qualitative des macrophytes sur la régulation hydrochimique du lac Tchad. Cah. ORSTOM, ser. Hydrobio., 12 (1), 65-69.

12. César, J. \& Chatelain, C. (2019). Flore illustrée du Tchad. Conservatoire et Jardin botanique de Genève en co-édition avec l'Université de N'Djaména et la Coopération Suisse au Tchad, 767 p.

13. Chauvin, C., Bertrin, C., Boutry, S., Feret, T., Loriot, S. \& Dutartre, A. (2014). Des méthodes basées sur les peuplements de macrophytes pour évaluer l'état écologique des milieux aquatiques ; Sciences Eaux \& Territoires, 15, 54-59.

14. Dajoz, R. (2006). Précis d'écologie. 8e édition Dunod, Paris, 434 p.

15. De Villers, J., Squilbin, M. \& Yourassowsky, C. (2005). Qualité physico-chimique et chimique des eaux de surface : cadre général Institut Bruxellois pour la Gestion de l'Environnement / Observatoire des Données de l'Environnement. 16 p.

16. Dimon, F., Dovonou, F., Adjahossou, N., Chouti, W., Mama, D., Alassane, A. \& Boukari M. (2014). Caractérisation physico-chimique du lac Ahémé (Sud Bénin) et mise en relief de la pollution des sédiments par le plomb, le zinc et l'arsenic. J. Soc. Ouest-Afr. Chim. 37 ; 36-42

17. FAO (2002). Luttes contre les principaux végétaux aquatiques envahissants en Afrique. Activités et succès de la FAO 1991-2001.28p.

18. Fazul, A., Rachiq, S., Mikou, K. \& Saidomar, S. H. (2013). Contribution à l'étude de la flore aquatique d'un lac de montagne : Lac Dayet Aoua (Maroc). Afrique SCIENCE 09(3) 103-112.

19. Fetnaci, I., Beddiar, A. \& Hamel, T. (2019). Le lac Fetzara (Nord-Est algérien): Biodiversité floristique et menaces potentielles. Fl. Medit. $29: 227-245$

20. Frontier, S., (1983). Stratégies d'échantillonnage en écologie. Masson, Paris, $494 \mathrm{p}$

21. Gillet, F., De Foucault, B. \& Julve, P. (1991). La phytosociologie synusiale intégrée : objets et concepts. Candollea, 46, p 315 -340.

22. Grall, J. \& Coic, N. (2005). Synthèse des méthodes d'évaluation de la qualité du benthos en milieu côtier. éd. Ifremer, $91 \mathrm{p}$.

23. Hill, M. O. \& Gauch, H. G. Jr. (1980). Detrended correspondence analysis: an improved ordination technique. Vegetatio, 42, 47-58.

24. Houma, F., Belkessa, R., Khouider, A., Bachari, N. \& Derriche, Z. (2004). Étude corrélative des paramètres physico-chimiques et des données satellites IRS1C pour caractériser la pollution aquatique. Application à la baie d'Oran, Algérie. Revue des sciences de l'eau / Journal of Water Science, 17 (4), 429-446. 
25. Iltis, A. \& Lemoalle, J. (1979). La végétation aquatique du lac Tchad. ORSTOM ; Réunion de Travail sur la Limnologie Africaine, Nairobi (Kenya), 17 p.

26. Jouret, B. (1972). La méthode du transect appliquée à l'analyse urbaine. Un exemple bruxellois. Revue de géographie de Lyon, 47 (1), 77-96.

27. Kadid, Y., Thébaud, G., Pétel, G. \& Abdelkrim, H. (2007). Les communautés végétales aquatiques de la classe des Potametea du lac Tonga, El-Kala, Algérie, Acta Botanica Gallica, 154 : 4, 597-618,

28. Lacroix, G. \& Danger, M. (2008). Des réseaux trophiques au fonctionnement des écosystèmes lacustres : vers une intégration de l'hétérogénéité et de la complexité. Revue des sciences de l'eau / Journal of Water Science, 21 (2), 155-172.

29. Lavoie, C. (2012). Le roseau commun au Québec : enquête sur une invasion. Le naturaliste canadien ; 136 No 3 ; 33-39.

30. Lebrun, J. (1947). La végétation de la plaine alluviale au sud du lac Edouard. Institut des Parcs Nationaux du Congo Belge, Bruxelles, Fascicule 1, 391 p.

31. Lemoalle, J., Bader, J-C., Leblanc, M. \& Sedick A. (2012). Recent changes in Lake Chad : observations, simulations and management options (1973-2011), Global and Planetary Change 80-81 : 247-254.

32. Léonard, J., (1974). Aperçu sur la végétation de la partie est du lac Tchad. ORSTOM, N'Djaména, 14 p.

33. Les, D. H. (1986). The phytogeography of Ceratophyllum demersum and C. echinatum (Cerathophyllaceae) in glaciated North America. Can. J. Bot. 64: 498 - 509.

34. Mikail, A., Karar, M., Tidjani, A., Mahonte, S., Brahim, B.O. \& Laleye, P. (2018). Impact of Intensification of Agropastoral Activities on the Water Quality of Lake Fitri, International Journal of Sciences, vol 7, 66-73.

35. Montegut, J., (1987). Le milieu aquatique : Tome1: Milieu aquatique et flore. Paris : ACTA, $60 \mathrm{p}$.

36. Moustapha, A. M., Sanoussi, A., Sani, L. M. \& Alfazazi, Y. (2012). Traitement des eaux usées par lagunage : Evaluation de la production et du pouvoir épurateur du bourgou (Echinochloa stagnina). Bulletin de la Recherche Agronomique du Bénin (BRAB) Numéro spécial Productions Végétales \& Animales et Economie \& Sociologie Rurales. 47-55.

37. Müller, J.V. \& Wezel, A. (2006). Inundated grasslands with Echinochloa colona (L.) Link in semi-arid and sub-humid West Africa. Belgian Journal of Botany 139 (2): 203-219. 
38. Ndour, S., Mbaye, M. S., Diouf, J., Mballo, R., Sarr, M., Gueye, M. \& Noba K. (2019). Impacts écologiques et socio-économiques de Ceratophyllum demersum L., une plante aquatique envahissante dans le delta du fleuve Sénégal, Int. J. Biol. Chem. Sci. 13(6): 2739-2749.

39. Noumon, C. J., Mama, D., Dedjiho, C. A., Agbossou, E. \& Ibouraima, S. (2015). Evaluation

40. de la qualité physico-chimique et du risque d'eutrophisation de la retenue d'eau de

41. Kogbétohouè (Sud-Bénin), Journal of Applied Biosciences, 85, 7848 $-7861$.

42. OFEV (2009). Méthodes d'analyse et d'appréciation des cours d'eau. Macrophytes : Instructions pour le prélèvement d'échantillons. Office fédéral de l'environnement, Berne : $60 \mathrm{p}$.

43. Paigbc, 2012. Plan d'aménagement intégré à base communautaire, site de Boum kébir. Ministère de l'Environnement et des Ressources halieutiques, direction des pêches et de l'aquaculture, $110 \mathrm{p}$.

44. Pias, J. (1970). La végétation du Tchad : ses rapports avec le sol. Variations paléobotaniques au quaternaire. Contribution à la connaissance du bassin tchadien. ORSTOM, $\mathrm{n}^{\circ} 6$. Paris, $49 \mathrm{p}$.

45. Pias, J. \& Barbery J. (1965). Cartes pédologiques de reconnaissance au 1/200 000 : feuilles de Lac Iro - Djouna. Paris : ORSTOM, (25), 99 p. (Notice Explicative ; 25).

46. Potères, R. (1950). Compétition au sein de groupements végétaux aquatiques dans les lagunes de la Côte d'Ivoire, Bulletin de la Société de Botanique de France, 97 : 4-6, 102-112.

47. Robyns, W. (1936). Contribution à l'étude des formations herbeuses du district forestier central du Congo Belge. Mem. Inst Roy Col Belge Section Méd (5), 151 p.

48. Saradoum, G., Guissé, A., Diallo, A. \& Faye, M. N. (2012). Characterization of herbaceous vegetation of the National Parc of Manda in Chad. International Journal of Science and Advanced Technology, 2 (6) 1-10.

49. Sarr, A., Thiam, A. \& Bâ, A. T. (2001). Macrophytes et groupements végétaux aquatiques et amphibies de la basse vallée du Ferlo (Sénégal). African Journal of Science and Technology (AJST). Science and Engineering Series Vol. 2, No. 1, pp. 89-97

50. Saunier, M., Raimond, C. \& Amba, B. A., (2017). Les « territoires d'eau » ou comment gérer les ressources halieutiques dans les espaces lacustres : les cas du lac Fitri et Iro. Colloque international : « Recherches croisées sur les écosystèmes lacustres tchadiens » 8-33. 
51. Syed, I., Fatima, H., Mohammed, A. \& Siddiqui, M. A. (2018) : Ceratophyllum demersum a Free-floating Aquatic Plant. Indian J.Pharm.Biol.Res.; 6(2):10-17.

52. Taffouo V. D., Saya R. A. I., Mbeng L. O. \& Eyango M. T. (2017). Impacts des caractéristiques physico-chimiques des eaux sur la distribution du phytoplancton et des macrophytes de la rivière Nkam (Cameroun). Int. J. Biol. Chem. Sci. 11(4): 1766-1784

53. Thiam, M. A. (2012). Etude de la flore vasculaire, de la végétation et des macrophytes aquatiques proliférants dans le Delta du fleuve Sénégal et le Lac des Guiers (Sénégal). Université Cheikh Anta Diop de Dakar, Faculté des Sciences et Techniques (Sénégal). 240 p.

54. Vandhen Berghen, C. (1988). Flore illustrée du Sénégal. Gouvernement du Sénégal, Ministère du Développement rural, Direction des Eaux et Forêts, Dakar, tome II, p 412.

55. Wiegleb, G. (1988). Analysis of Flora and Vegetation in Rivers: Concepts and Applications. In: Symoens J.J. (eds) Vegetation of inland waters. Handbook of vegetation science, vol 15-1. 311-340. 